\title{
UNIVERSITY OF CALIFORNIA
}
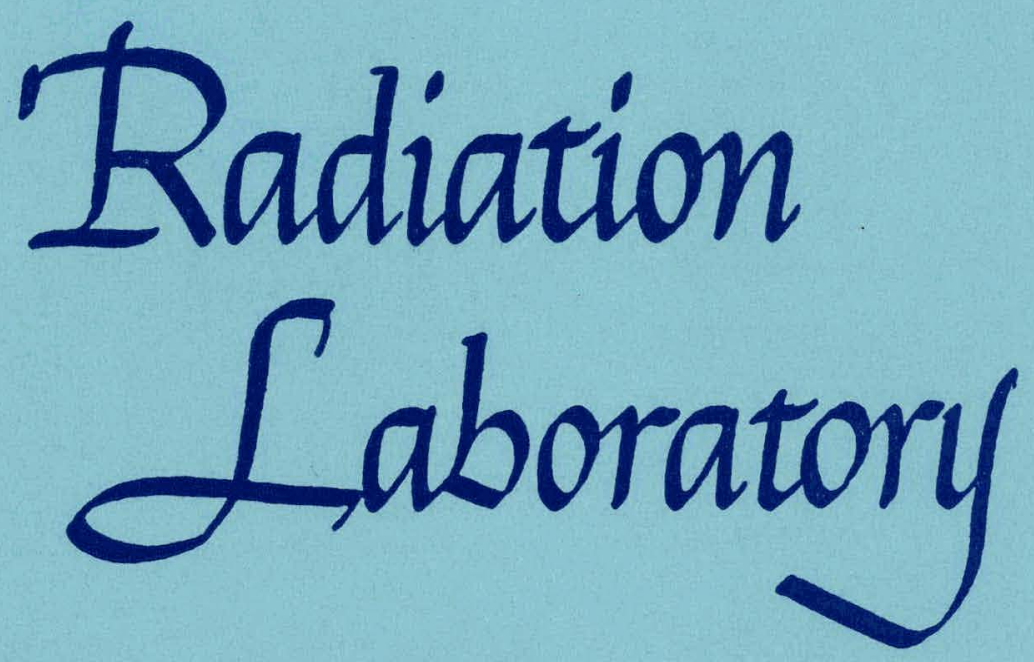

SEMI-EMPIRICAL CORRELATIONS OF ALPHA DECAY RATES AND ENERGIES

BERKELEY, CALIFORNIA 


\section{DISCLAIMER}

This report was prepared as an account of work sponsored by an agency of the United States Government. Neither the United States Government nor any agency Thereof, nor any of their employees, makes any warranty, express or implied, or assumes any legal liability or responsibility for the accuracy, completeness, or usefulness of any information, apparatus, product, or process disclosed, or represents that its use would not infringe privately owned rights. Reference herein to any specific commercial product, process, or service by trade name, trademark, manufacturer, or otherwise does not necessarily constitute or imply its endorsement, recommendation, or favoring by the United States Government or any agency thereof. The views and opinions of authors expressed herein do not necessarily state or reflect those of the United States Government or any agency thereof. 


\section{DISCLAIMER}

Portions of this document may be illegible in electronic image products. Images are produced from the best available original document. 
$\perp$

UCRL- 3176

Chemistry Distribution

\section{UNIVERSITY OF CALIFORNIA}

Radiation Laboratory

Berkeley, California

Contract No. W-7405-eng-48

SEMI-EMPIRICAL CORRELATIONS OF

ALPHA DECAY RATES AND ENERGIES

Charles J. Gallagher, Jr: and John O. Rasmussen

October 26, 1955

Printed for the U. S. Atomic Energy Commission 


\title{
SEMI-EMPIRICAL CORRELATIONS OF ALPHA DECAY. RATES AND ENERGIES
}

Charles J. Gallagher, Jr. and John O. Rasmussen Radiation Laboratory and Department of Chemistry University of California, Berkeley, California

\author{
October 26, 1955
}

\section{INTRODUCTION}

. Theoretical expressions relating alpha decay rate and energy were first derived by. Gamow ${ }^{1}$ and Gurney and Condon. ${ }^{2}$ The relationships hold best for even-even alpha emitters. Perlman, Seaborg, and Ghiorso 3 in their alpha systematics have pointed out large and erratic hindrance in odd types. Nordstrom ${ }^{4}$ has plotted distributions of hindrance factors in an attempt to compare "forbiddenness" in alpha decay in a manner similar to log ft values in beta decay. We have made hindrance factor. plots for all observed alpha groups emitted from nuclei with greater than:128 neutrons in an attempt to establish correlations.

\section{BASIS OF CALCULATIONS}

\section{Even-Even Nuclei - Ground State Transitions}

Decay rates to ground states of even-even nuclei are correlated remarkably well by simple barrier penetration formulas with smoothly. varying radius formulas. However, there are significant trends 5 as shown in a plot of "effective nuclear radius" for even-even alpha emitters. Thus, for our semi-empirical correlations of alpha decay rates we have chosen to plot decay rate trends of even-even nuclei on a type of plot which from barrier penetration theory should lead to nearly straight line plots for isotopes of a giver element. The type 
of plot chosen sets the logarithm of the half-life against the reciprocal of the square root of the total alpha decay energy. (We have included recoil energy and the electron screening correction:).

From this relationship between $\log t_{1 / 2}$ and $\mathrm{E}^{-1 / 2}$ the "hindrance" of an alpha particle is seen as the deviation from the curves. The alpha decay energy is then taken as the independent variable, and the hindrance factor is defined as the ratio of the experjmental partial a.7. pha half $\mathrm{mife}$ to that predicted from the curves.

The primary problem in this correlation, therefore, was to determine the lines which would serve as the basis for the calculations. This was done by the method of weighted least squares analyses on the ground state to ground state decay of even-even nuclei. The solid lines in. Fig. I are the result of these analyses.

The weighting consisted of giving spectroscopically determined alpha energies double weight as compared to ion chamber energy measurements. The solid triangles in Fig. 1 indicate the isotopes used in fitting the line. Solid circles indicate isotopes for which the halflives had been estimated from previous alpha systematics ${ }^{3}$ (i.e. $\operatorname{En}^{216}$, $\mathrm{Ra}^{220}$, $\mathrm{Th}^{224}$, etc.) or which appeared to deviate markedly from systematic behavior $\left(\mathrm{Po}^{212}, \mathrm{U}^{228}, \mathrm{U}^{238}\right.$ ) and these were not usen in the least squares analyses...For the elements up to curium there was: no problem In fltting the lines. for curium and higher elements the scarcity of: data made the fitting: somewhat arbitrary. . $\mathrm{Cm}^{242}$ and $\mathrm{Cm}^{244}$ were used exclusively to set the curium line because of their well determined decay schemes, as compared with the lighter curium isotopes: about... which insufficient experimental data is available. 
$\mathrm{Cf}^{250}$ and $\mathrm{Cf}^{252}$ were rejected in the determination of the californium line slopet because of their proximity to the closed subshell at 152 neutrons. 6 . The slope for fermium was obtained by extrapolation as indicated in Fig. 2 .

Alpha particle energies were obtained from the compilation of Perlman and Asaro ${ }^{5}$ with the exception of the isotopes listed below. All partial alpha half-lives were obtained using half-lives, alpha branching ratios, and alpha particle abundances listed in the Table of. Isotopes ${ }^{2}$ except for the following: $\mathrm{Em}^{204}$, $\mathrm{Em}^{206}$, Reference 8; $\operatorname{Ra}^{222}, \alpha_{1}$, Reference $.9 ; \mathrm{U}^{230}, \alpha_{2}$, Reference 10; $\mathrm{U}^{232}, \alpha_{3}$, Reference $11 ; \mathrm{Np}^{237}$, all states, Reference $12 ; \mathrm{Pu}^{238}, \alpha_{3}, \alpha_{4}$, Reference $13 ; \mathrm{Am}^{243}, \alpha_{3}, \alpha_{4}$, Reference $14 ; \mathrm{Cm}^{242}, \alpha_{3}, \alpha_{4}, \alpha_{5}$, Reference 15; $\mathrm{Cm}^{243}, \alpha_{1}$, Reference $16 ; \mathrm{cm}^{245}, \alpha_{0}$, Reference $17 ;$ $\mathrm{Cf}^{246}, \alpha_{2}, \alpha_{3}$, Reference 1ـ8; $\mathrm{Cf}^{249}, \alpha_{0}, \alpha_{1}$, Reference 19; $\mathrm{Cf}^{250}$, $\alpha_{0}, \alpha_{1}$, Reference $20 ; \mathrm{Cf}^{252}, \alpha_{0}, \alpha_{1}$, Reference $21 ; \mathrm{E}^{253}, \alpha_{0}, \alpha_{1}$, $\alpha_{2}$, Reference $2 \dot{\xi} ; \mathrm{E}^{254}, \alpha_{0}$, Reference $23 ; \mathrm{Fm}^{254}, \alpha_{0}, \dot{\alpha}_{1}, \alpha_{2}$; Reference 24 .

Wince this paper was prepared, new data have been obtained on the lighter californium isotopes which reassign the alpha decay energy and half-life of $\mathrm{Cf}^{244}$ to $\mathrm{Cf}^{245}$ and which give the actual half-life and alpha particle energy of $\mathrm{Cf}^{244}$ (25) Since these new data have been found to alter the slope of the $\mathrm{cf}$ line only slightly due to the anomalous behavior of $\mathrm{Cf}^{245}$, we have not recalculated the hindrance factors for the isotopes of berkelium, californium, einsteinium, and fermium which would be affected if the change were large. 
It is easy to justify the above-mentioned type of plot from WKB barrier penetration formulas (the form for S-wave emission). The most important factor in these formulas is the exponential expression

$$
P=\exp \left[-\frac{2}{2} \int_{R}^{E^{2}}\left[2 M\left(\frac{2 Z e^{2}}{r}-E\right)\right]^{I / 2} d r\right]
$$

which is expressible in analytical form as

$$
\exp \left[-\frac{2}{\hbar}(\operatorname{PMB})^{1 / 2} \mathrm{R}\right]\left[\mathrm{x}^{-1 / 2} \operatorname{arc} \cos \mathrm{x}^{1 / 2}=(1-\mathrm{x})^{1 / 2}\right]
$$

where $x .=E R / 2 Z e^{2}$, a dimensionless parameter, and $B=2 Z e^{2} / R$, the barrier height. If we make a. Taylor series expansion of this function about $\mathrm{x}=0$, we obtain

$$
P \approx \exp \left\{-\frac{(2 M)^{I / Z_{B} I / 2_{R}}}{\hbar}\left[\frac{\pi B^{I / 2}}{E^{I / 2}}-4+\frac{E}{B}-\frac{3}{4} \cdot \frac{E^{2}}{B^{2}}+\ldots\right]\right\}
$$

If one retains only the dominant first two terms, as has Gamow ${ }^{l}$, then one sees that the logarithm of the penetration function is a linear function Of $\mathrm{E}^{-1 / 2}$.

An interesting check on simple barrier penetration theory is provided by examination of the slopes of the least squares lines of Fig. 1. The form of the first term of Equation (3) shows that the slopes of the lines of Fig. I should be proportional to the atomic number, $Z$, of the daughter nucleus to accord with simple theory. In Fig. 2 these slopes are plotted against $\mathrm{Z}$. The theoretically expected behavior is well shown by the lower elements in the series, but there is a striking break beginning at the plutonium alpha emitters and then a reversal of the expected trend. 
It is beyond the scope of this paper to attempt any detailed theoretical explanation of the deviations from simple theory. It should be pointed out that abnormal slopes in Fig. I do not necessarily mean a changed dependence of decay rate on energy, but since energy is (above 128 neutrons) amonotonic function of the neutron number for an isotopic series, an abnormal slope may reflect a change in the alpha decay rate dependent on the addition of neutron pairs. Some isotopic series pass through the region of the onset of validity of the Bohr-Mottelson 26 spheroidally deformed model and some pass through rapidly changing spheroidal deformation. Such changes must certainly influence barrier penetration, yet, interestingly enough, the elements where these changes are greatest, i.e. radium, thorium, and uranium, have the best-behaved slopes. The slopes should also be influenced by variations in the alpha formation probability entirely apart from the external barrier penetration factor.

\section{Even-Even Nuclei - Excited State Transitions}

In contrast to the regularly behaving ground state transition rates, the transitions to excited states show striking trends in rate for different nuclei.

For the elements thorium and above, alpha decay is of ten observed to at least three levels $(0+, 2+$, and $4+)$, identifiable as a rotational band sequence. Alpha decay to states of odd parity has been observed? To exhibit this behavior we have calculated hindrance factors to all observed states. The distribution of hindrance factors is shown in the histograms of f'igures $3,4,5$, and 6 . The three lines of numbersion 
each histogram block represents (1) element/mass number/ground state, indicated by zero if this is the ground state, (2) assigned number of excited state and energy of this state in kilo-electron volts, and (3) the value of the departure factor for this transition. Because of the increasing interest in the correlations achieved by the Bohr-Mottelson . large-deformation model, we have segregated the cases in the region of nuclear rotational bands from those cases nearer the $\mathrm{Pb}{ }^{208}$ closed cont'iguration. The bold line on each histogram divides these regione, the rases belew the bold line coming from the robluluplat kund region.

For the even-even nuclides the generally increasing nature of Hindrance to the higher groups is evident, and the hindrance to the 4+, $6+$, and $8+$ groups generally far exceeds hindrance accountable for by the addition to the barrier of the centrifugal potential associated with angular momentum of the alpha decay system.

Asaro $^{27}$ has plutted hindrance factors* against atomic number for the even parity excited state transitions. In somewhat similar manner we have plotted hindrance factors against neutron number in Fig. 7 .

*The hindrance factors of Asaro for transitions to excited sta.tes of even-even nuclei are defined slightly differently from ours. Asaro has taken as unhindered the ground state to ground state even-even alpha transitions. We have, however, calculated hindrance factors in relation to the least square curves in Fig. 1: Consequently, we may have hindrance factors for ground state trancitions, whereas Asaro will not. The O+ hindrance factors shown in Fig. 7 illustrate this and can be used to obtain Asaro type hindrance factors from the present data. 
We do not expect to illustrate any trends with the $0+$ hindrances, but the high hinỏrances of the lightest isotopes of polonium, emanation, and plutonium and the slight depression at 142 neutrons are of interest. The variation observed for the curium and californium isotopes is explained by the manner, previously mentioned, in which the lines for these elements in Fig. I were determined.

The dependence upon neutron number of decay to the first excited state $(2+)$ reflects the ground state transition probability and appears relatively unhindered, although there is some increase among the heaviest isotopes.

Of very great interest is the dependence of decay to the second excited state (4+) upon $N$ and $Z$. It can easily be seen from Fig. 7(c) that the hindrance factors for the second state vary with $\mathrm{Z}$ and pass through a maximum at $\mathrm{Z}=96$. However, also notable is the apparent neutron dependence of isotopes, as manifested by the apparent maxima in the $4+$ curves of thorium and uranium. The 1- states apparently follow the behavior of the $4+$ states.

The relative intensities of decay groups to the even parity states have been considered by a number of authors in connection with spheroidal alpha decay theory but will not be discussed herè

\section{Odd-Nucleon Alpha Emitters}

In contrast to the regular behavior of even-even alpha decay rates, nuclei with odd nucleon numbers often exhibit erratic alpha decay rates, tending generally to be slower than comparable even-even alpha emitters. Perlman, Ghiorso, and Seaborg 3 have clearly pointed out that the hindrance must be associated with a lowered alpha formation probability rather than with the higher external barrier resulting from non-zero angular momentum. 
The details regarding this hindrance are not.well understood. Rasmussen 29 and Bohr, Froman, and Mottelson ${ }^{30}$ have selected and discussed a few special cases exhibiting little or no hindrance.

To obtain the bases for calculation of these hindrance factors for odd $Z$ nuclei the slopes shown in Fig. I wére 1ntérpolated to yield the intermediate odd $\mathrm{Z}$ slopes. For instance, the average of the slopes for elements 84 and 86 was employed as lhe slope for $z=83$. Since it was impossible.

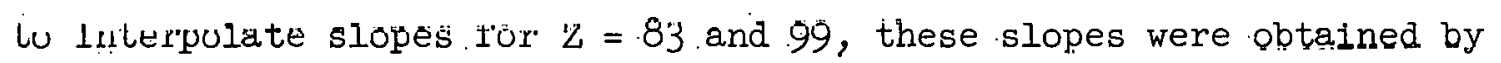
extrapolation, as indicated by the dashed line in Fig. 2. Similarly the intercepts at. $\mathrm{E}_{\alpha}=\infty$ were interpolated to yield the intercepts for these nuclei.

The distributions of these hindrance factors for the various nuclear types are shown in Fig. 8 (odd-even), Fig. 9 (even-odd), and Fig. 10 (odd-odd). The histograms for odd-even and even-odd types exhibit.broad distributions.with the suggestion of a breakup into two groups. The main group peaks around hindrance factors of order 3 , and the second and small group, around 500 .

For the odd-even types in the rotational band region, most of the cuses in the highest hindered group probably invoive a change in parity in alpha decay. This fact is.inferred from the apparent "favored" nature of some of the transition in the same nuclei, with the levelo showing the high hindrance connected to the favored levels by El gamma transitions, which signify a parity difference. Thus we suggest the possibility that parity change is a major hinderingfactor in alpha decay of odd mass nuclei. Examination of other odd-even alpha transitions possibly involving a parity change indicates that the alpha transition may generally be hindered in such cases but that the hindrance associated with the parity change becomes less at atomic numbers below 95. 
For the even-odd types in the rotational band region one again observed a separate group of hindered cases for log hindrance factor from 2.0 to 3.5. Here, however, we may infer that at least two cases (in the decay of $\mathrm{Cm}^{243}$ ) probably involve no parity change. This inference may be drawn from the probable presence of a "favored" alpha transition in $\mathrm{Cm}^{243}$ and the connection between favored and hindered states by. Mamma. '. transitions.

\section{CONCLUSION}

The data presented here represent all that are available at present on alpha decay rates. We have attempted, by presenting them in the form of hindrance factor histograms, to iliustrate the distribution of the deviations from simple theory. We hope that the presentation of the data in this form can serve as a useful reference for further studies.

\section{ACKNOWLEDGMENTSS : . .}

We are indebted to Dr. Frank Asaro and Dr. Frank Stephens and to John Hummel, Albert.Ghiorso, and others for valuable data in advance of. publication. This study was carried out under the auspices of the U.S. Atomic Energy Commission. 
1. G. Gamow, Z. Physik 51, 204 (1928); G. Gamow and.C. L. Critchfield, Theory of Atomic Nucleus and Nuclear Energy Sources (Clarendion Press; Oxford, 1949), Chapituer VI.

2. E. U. Condon and R. W. Gurney, Phys. Rev. 33, 127 (1929); Nature 122, $439(1928)$.

3. I. Perlman, G. T. Seaborg, and A. Ghiorso, Phys. Rev. 77, $26:(1950)$ :

1. S. G. Nordströn, Manne Siegbahn (Almquist and Wiksel. 1s Bntryckeri $A b$, Uppsala, . 1951), p. 587 :

5. I. Perlman and F. Asaro, Ann. Rev: Nuclear Sci. 4 (1954);: F. S. Stephens, Jr., private communication.

6. A. Ghiorso, S. G. Thompson, G. H. Higgins, B. G. Harvey, and G. T. Seaborg, Phys. Rev. 95, 293 (1954).

7. J. M. Hoilander, I. Perlman, and G. T. Seaborg, Revs. Modern ..Phys. 25; $469(1953)$.

8. F. Asaro and I.: Perlman, Revs. Modern Phys. 2., 456 (1954); 1 . Etoncr and E. K. Hyde, private communications.

9. J. M. Hollander, W. G. Smith, and F. Asaro, private communications.

10. F. S. Stephens, Jr.,.F. Asaro, and I. Perlman, Phys..Rev. 96,1568 (1954); F. Asaro and F. S. Stephens, Jr., private communications.

11. F. Asaro and I. Perlman, Phys. Rev. 22, 37. (1955); G. Bcliar.rr-Guldhaber, E. der Mateosian, G. Häarbottle, and M. McKeown, Phys. Rev. . 99, 180 (1955).

12. D. Engelkemeir and L. B. Magnussnn, private communication from T. Nuvey.

13. F. Asaro and I. Perlman, Phys. Rev. 94, 381 (1954); F. Asaro, private communication. 
14. F. S. Stephens, Jr., J. Hummel, F. Asaro, and I. Perlman, Phys. Rev. 98,261 (1955).

15. F. Asaro, S. G. Thompson, and I. Perlman; Phys. Rev. 92, 694 (1953);

F. Asaro, private communication.

16. F. Asaro and I. Perlman, unpublished data (1954):

17. J. P. Hummel, F. Asaro, and I. Perlman, unpublished data (1955).

18. J. P. HumneI, F.S. Stephens; Jr.; F. Asaro; A. Chetham-Strode, Jr.., and I. Perlman, Phys. Rev. 98,. 22 (1953)); F. Asaro, private communication.

19. A. Ghiorso, G. R. Choppin, and B. G. Harvey, private communication.

20. F. Asaro, F. S. Stephens, Jr., B. G. Harvey, and I. Perlman, private communicetion.

21. F. Asaro, F. S. Stephens, Jr., B. G. Harvey, and I. Perlman, "Complex Alpha aici Gamma spectra of $\mathrm{Cf}^{252}$," Phys. Rev. (to be published).

22. F. S. Stephens, Jr., J. Hummel, F. Asaro, G. R. Choppin, and I. Perlman, private communication.

23. B. G. Harvey, S. G. Thompson, G. R. Choppin, and A. Ghiorso, Phys. Rev. 99, $337(1955)$.

24. F. Asaro, F. S. Stephens, Jr., S. G. Thompson, and I. Perlman, Phys. Rev. 98,19 (i955)。

25. A. Chetham-Strode, G. R. Choppin, and B.G. Harvey, "Mass Assignment of the 44-Minute $\mathrm{Cf}^{245}$ and the New Isotope $\mathrm{Cf}^{244}$," Phys. Rev. (to be published).

26. A. Bohr and B. R. Mottelson, Kgl. Danske Vedenskab. Selskab; Mat.-fys. Medd. 27, No. 16 (1953).

27. F. S. Stephens, Jr., Ph.D. Thesis, University of California Radiation Laburatory Unclassified Report UCRL-2970 (1955). 
28. J. 0: Rasmussen, University of California Radiation .'Laboratory .Unclassified Report UCRL-2431 (1953); L. Dresner, Ph.D. Thesis, Princetoñ University (1955); R..F. Christy, Phys. Rev. 98, ZA7; 1205 (1955); J. O. Rasmussen and B. Segall, University, of California Radiation Laboratory Unclassified Report UCRL-3040 (1955), "Alpha Decay of Spheroidal Nuclei," Phys. Rev. (to be published)。 29. J. 0. Raomussen, Arkiv Fysik I, .185 (1953).

30. A. Behr, P. O. Trbman, änd B. R. Mottelson, Kgl. Danske Venenskạ.h. Selskab, Mat.-fys. Medd. 20, No. 10 (1955). 


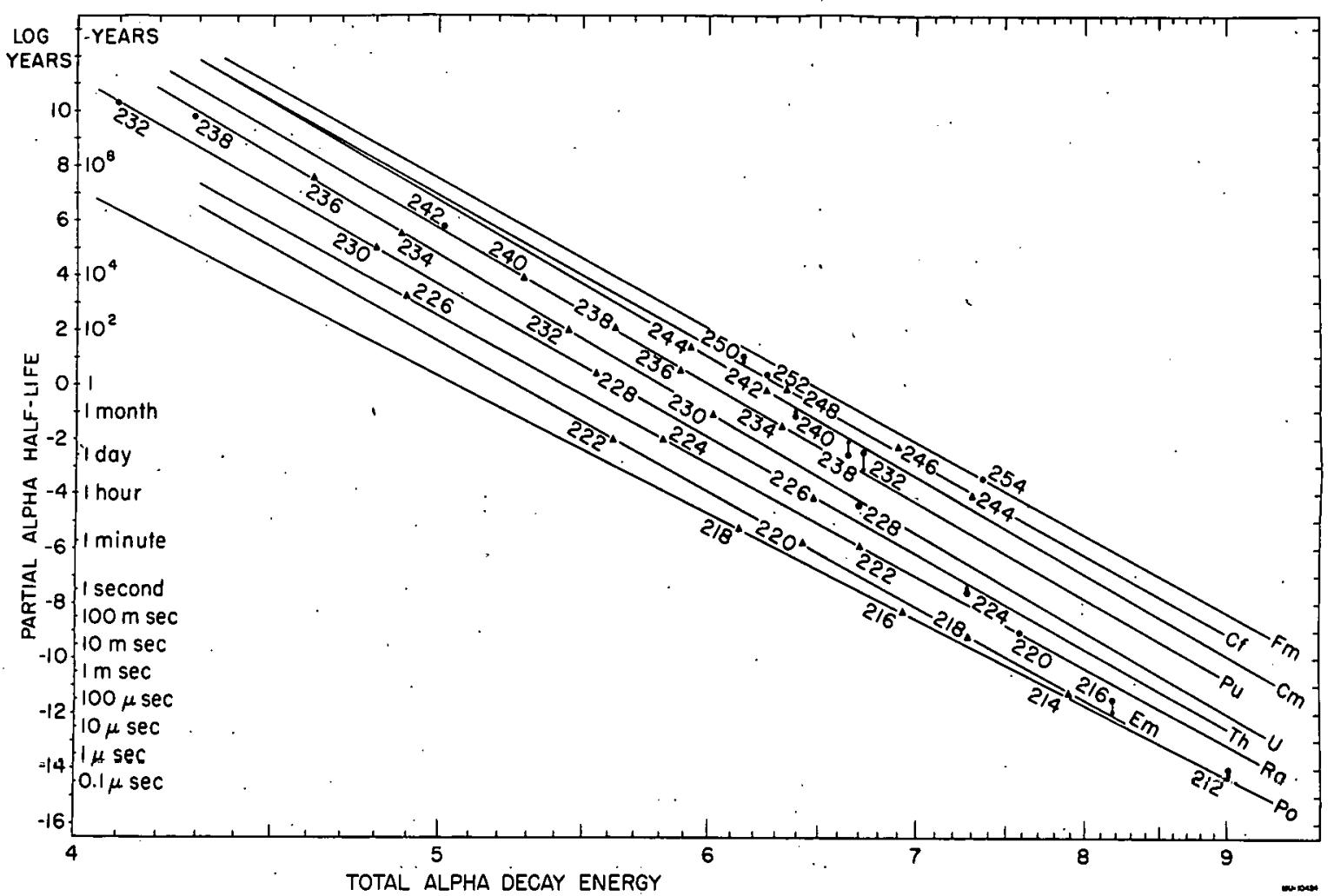

Fig. I Graph of log partial alpha half-life.vs. the reciprocal of the total alpha decay energy (alpha particle energy plus recoil and screening corrections). Solid lines represent least square fits to data represented by solid triangles, for $\mathrm{Z}$ between 84 and 98 . These least square fits were used to calculate hindrance factors. Eolid circles represent. data that were not used in the least squares analyses. 


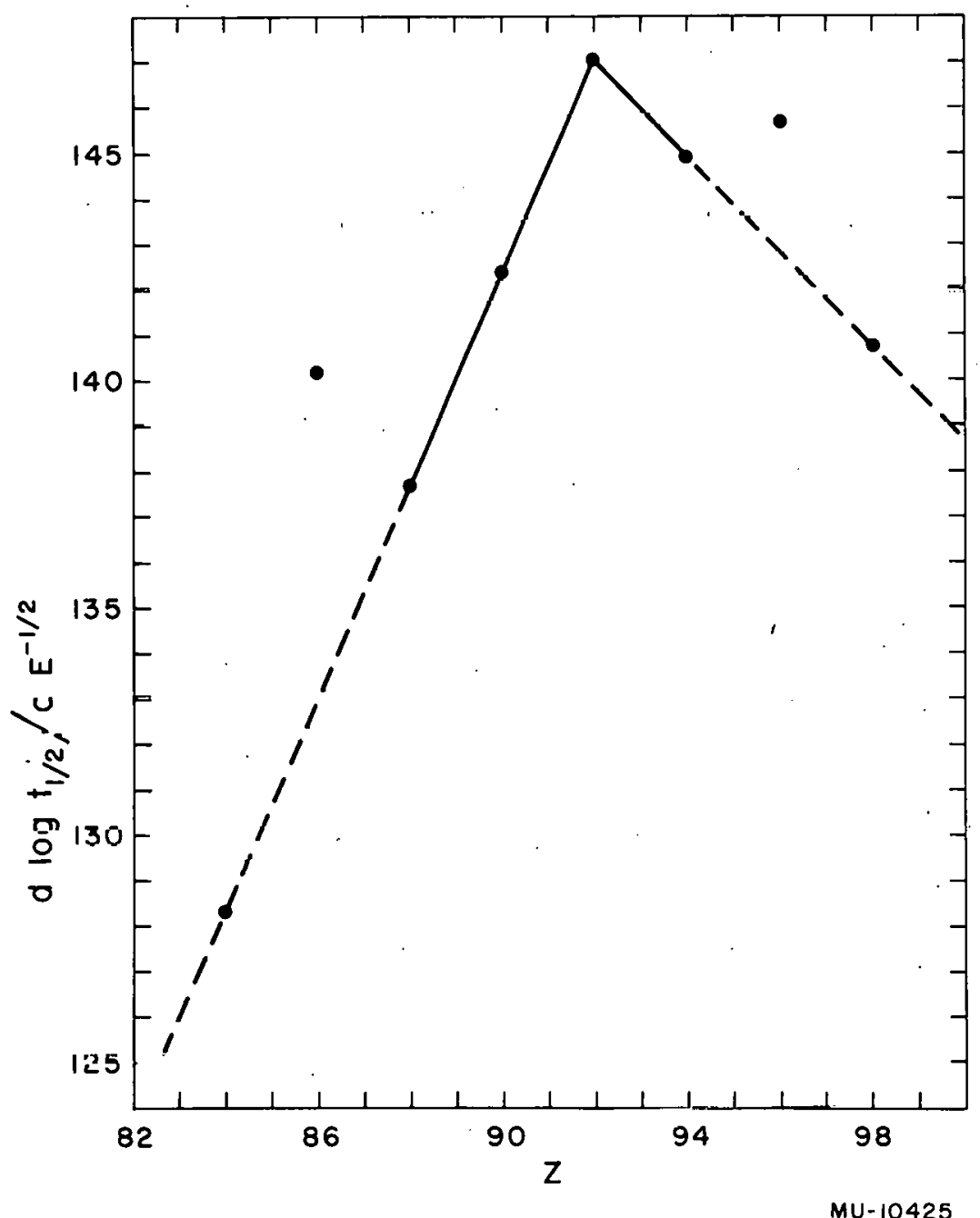

Fig. 2 Graph of $\frac{\mathrm{d} \log t / / \bar{c}}{d E^{-I / 2}}$ as a function of $\mathrm{z}$.

Dotted lines indicate method used to obtain slopes for elements 83,99 , and 100 . Irregularities after uranium probably indicate inadequacy of simple theory. 


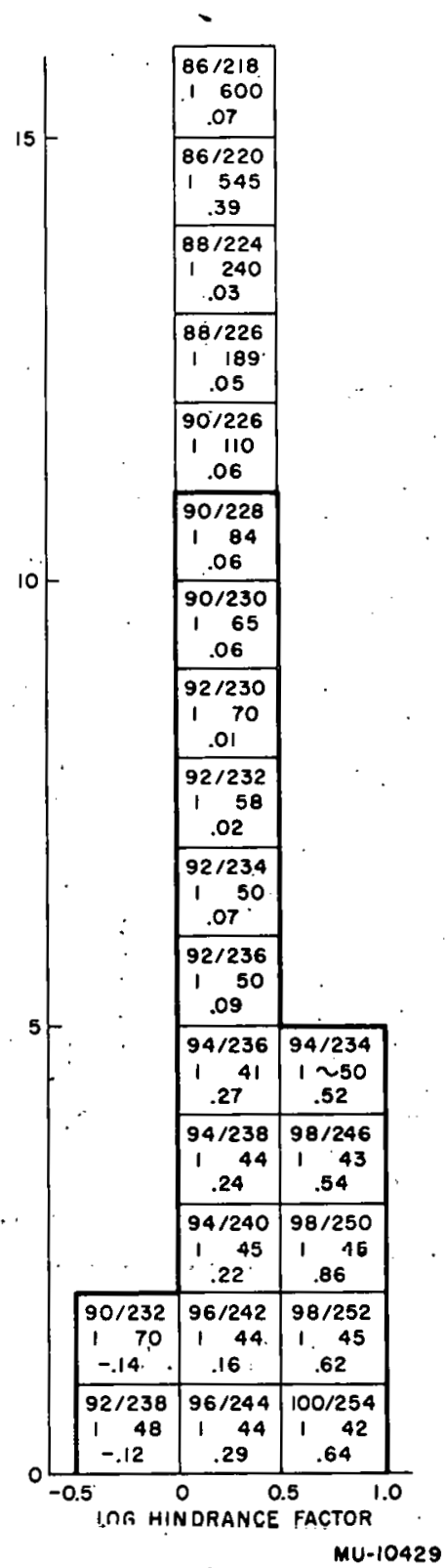

Fig. 3 Distribution of hindrance factors for alpha decay to $2+$ states of even-even nuclei. Heavy line in all histograms eeparates rotational region from region near closed shells, where rotational model is not applicalule. First line of numbers indicates element and mass assignment; second, present order of state and energy of state; third, value of $\mathrm{log}$ of hinarance factor. 


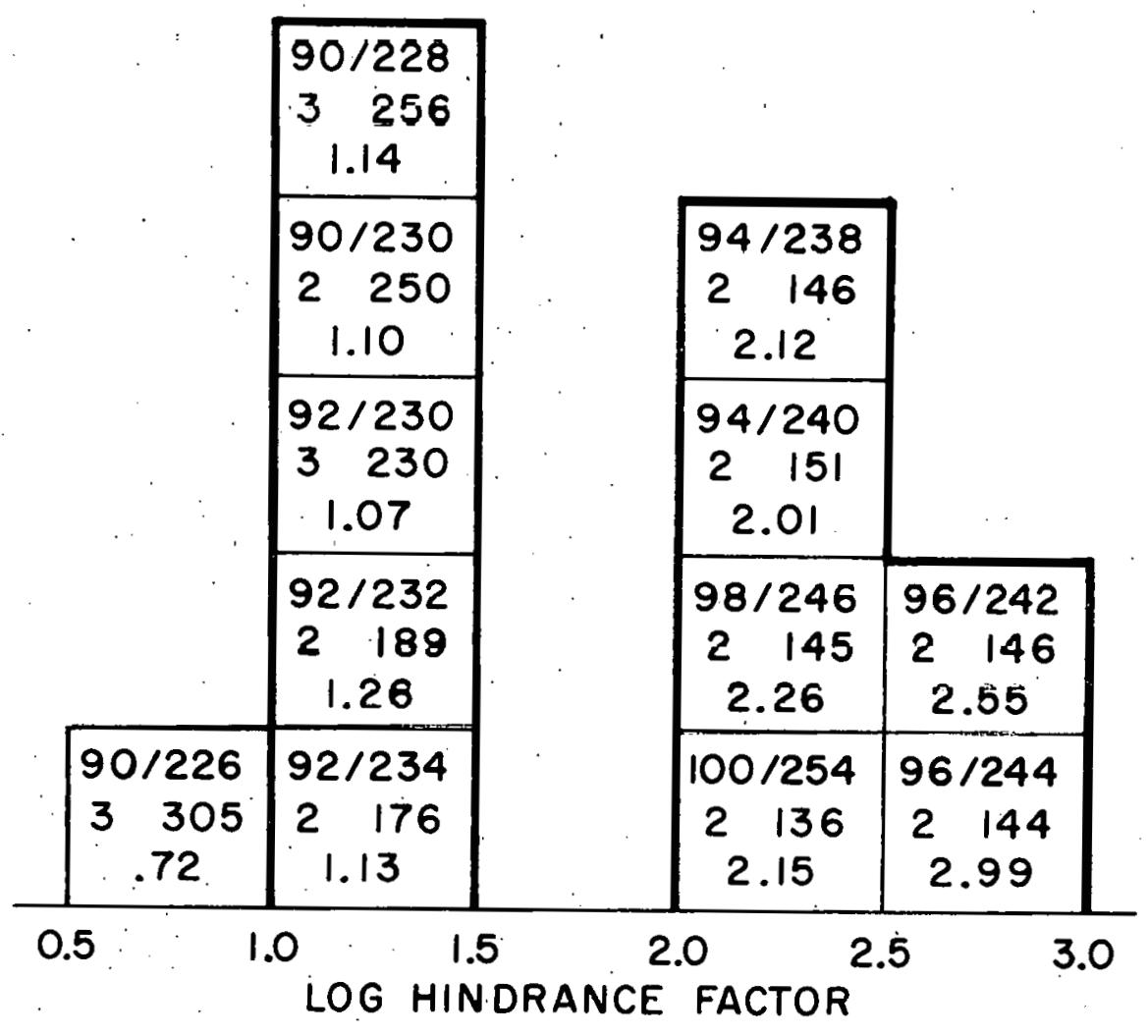

$M U=10427$

Fig. 4 Distribution of hindrance factors for alpha decay to $4+$ states in even-even nuclei. 


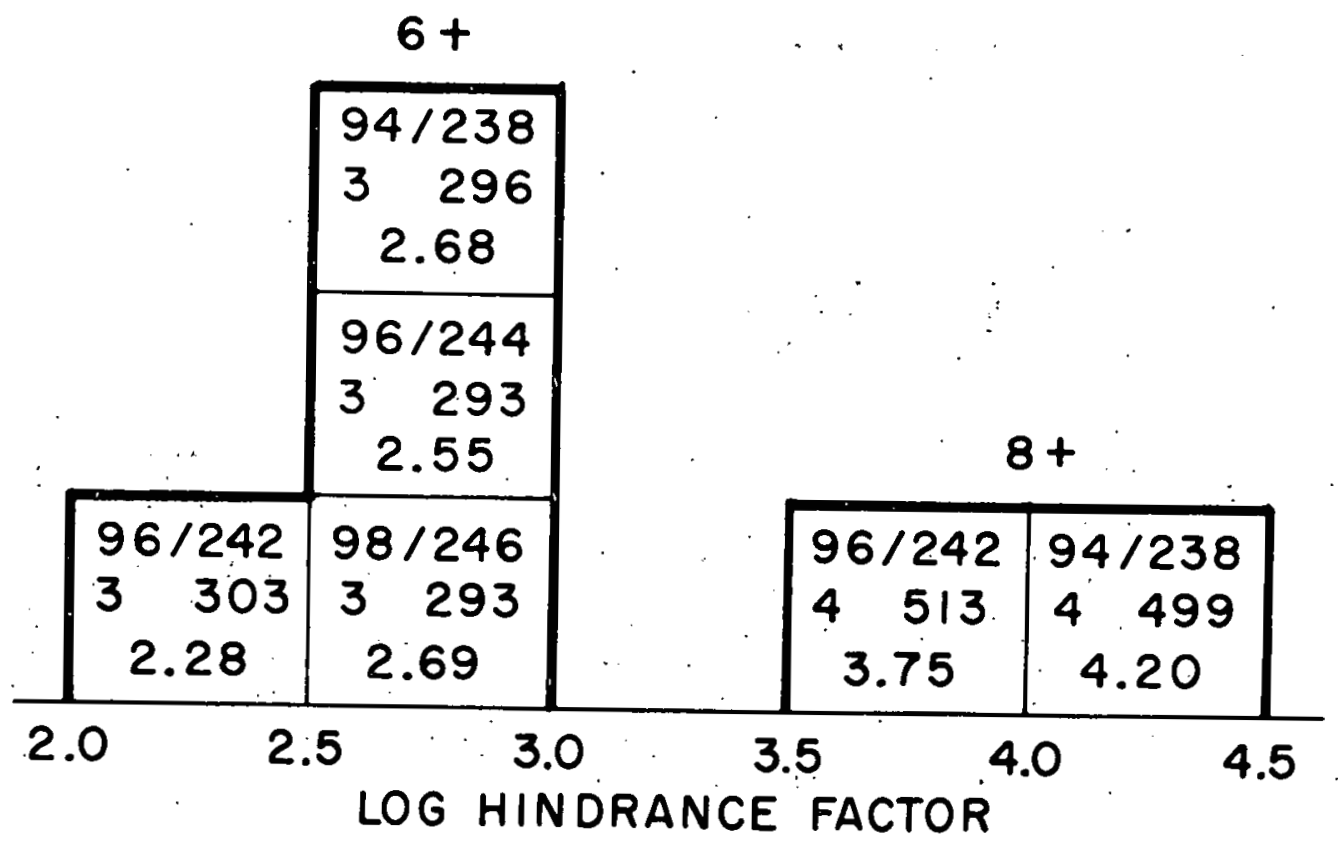

MU-10428

Fig. 5 Distribution of hindrance factors for alpha decay to 6+ and 8+ states in eveneven nuclei. 


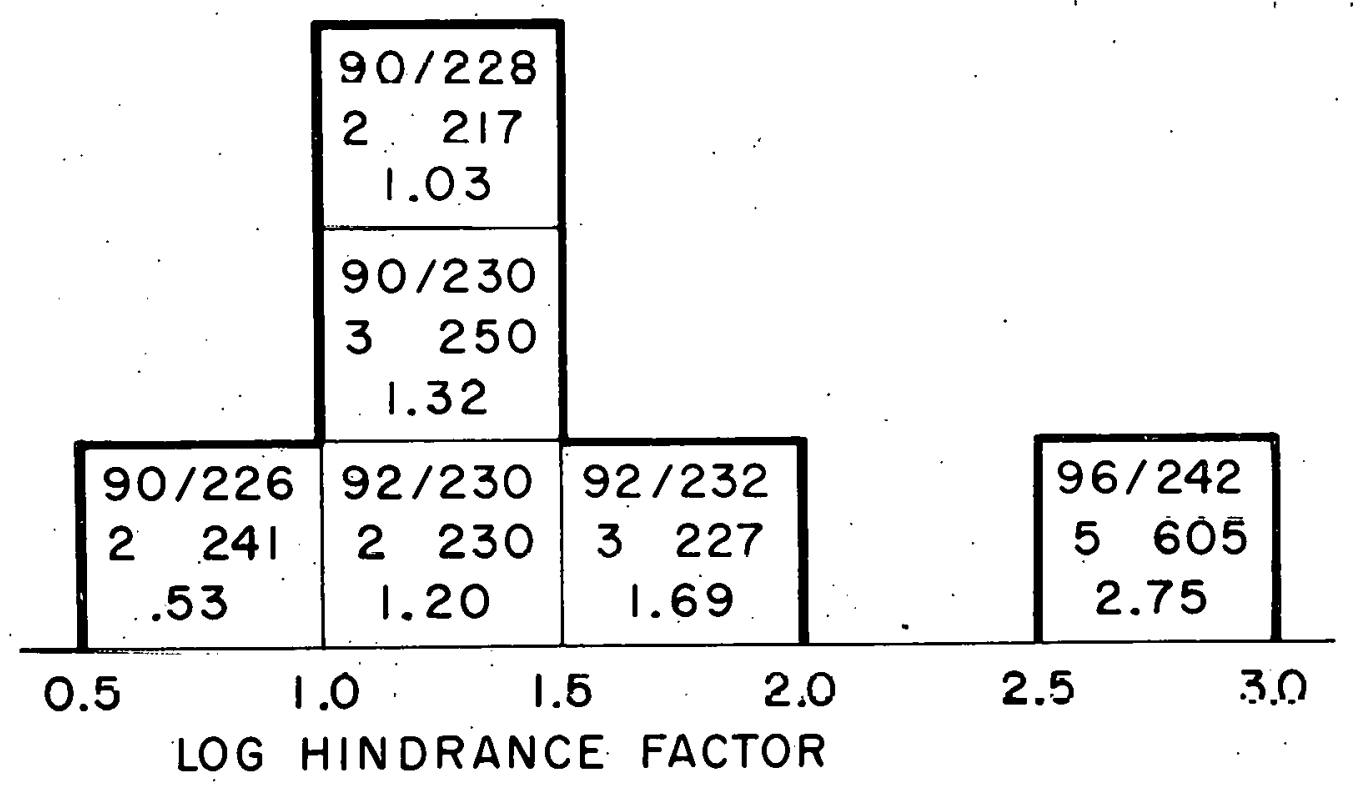

$M U-10426$

Fig: 6 Distribution of hindrance factors for alpha decay to 1 - states in even-even ruclei. 


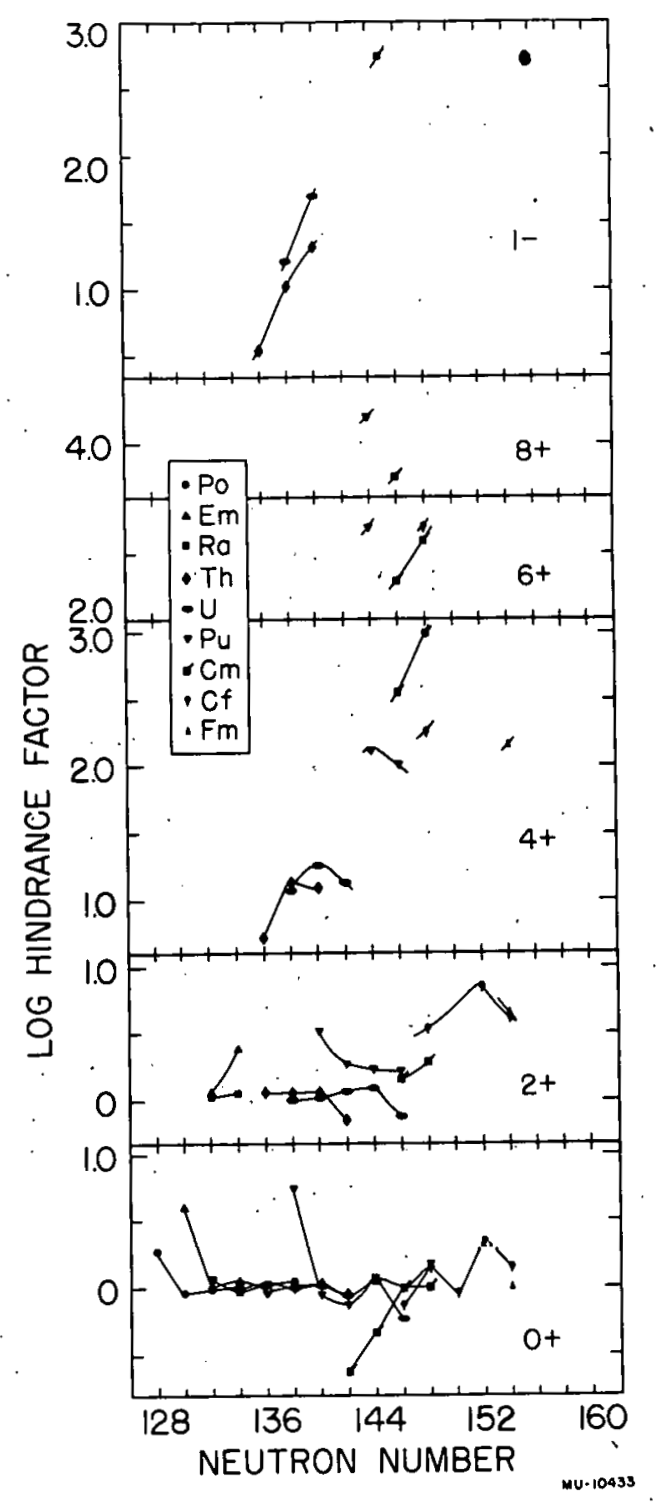

Fig. 7 Hindrance as a function of neutron number for decay to excited states in even-even nuclei. Lines connect isotopes. O+ is included as a basis for comparison with hindrance factors of Asaro. 


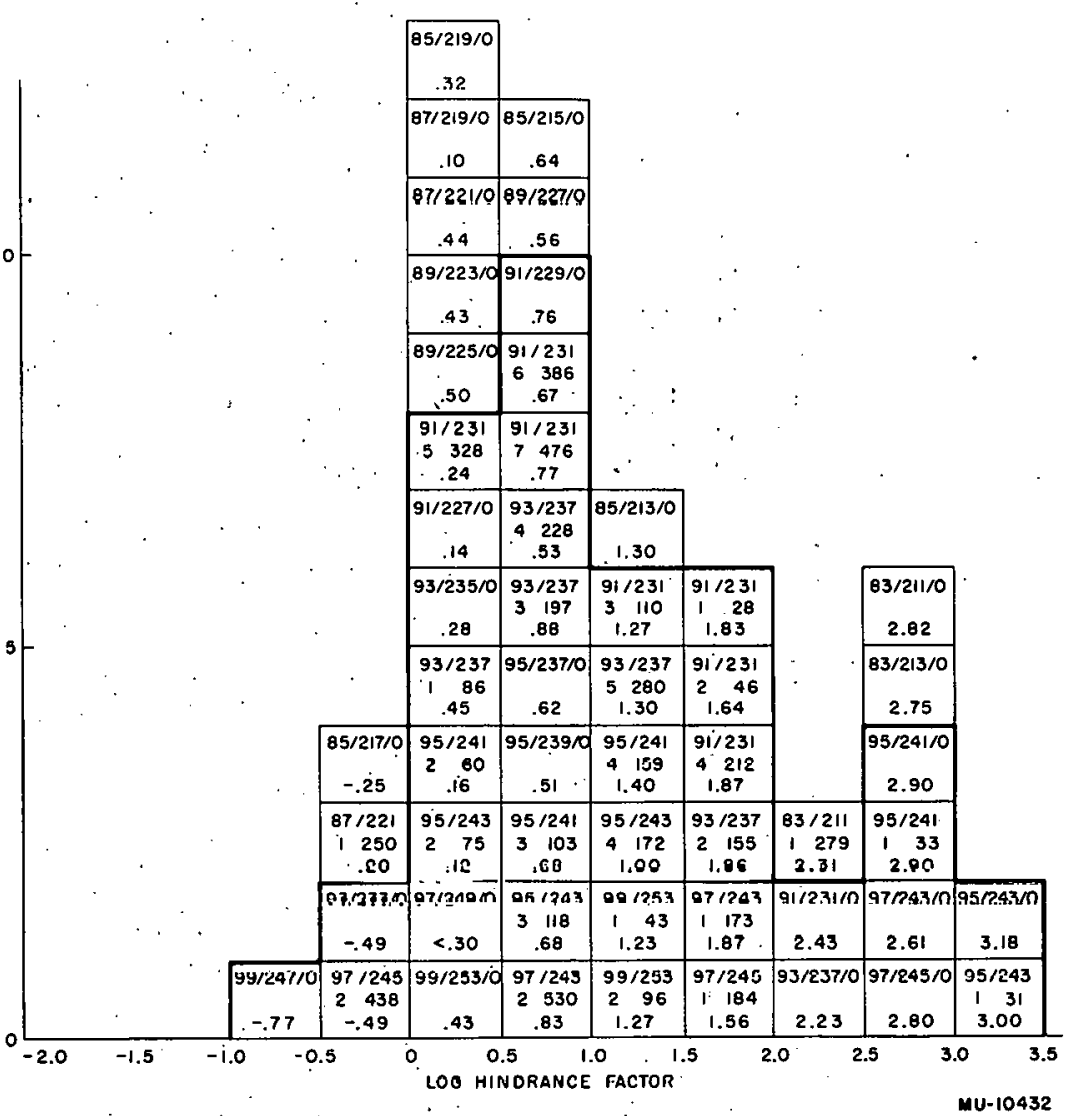

Fig. 8 Distribution of hindrance factors, for alpha decay for all observed cases to : states in even-odd nuclei: 


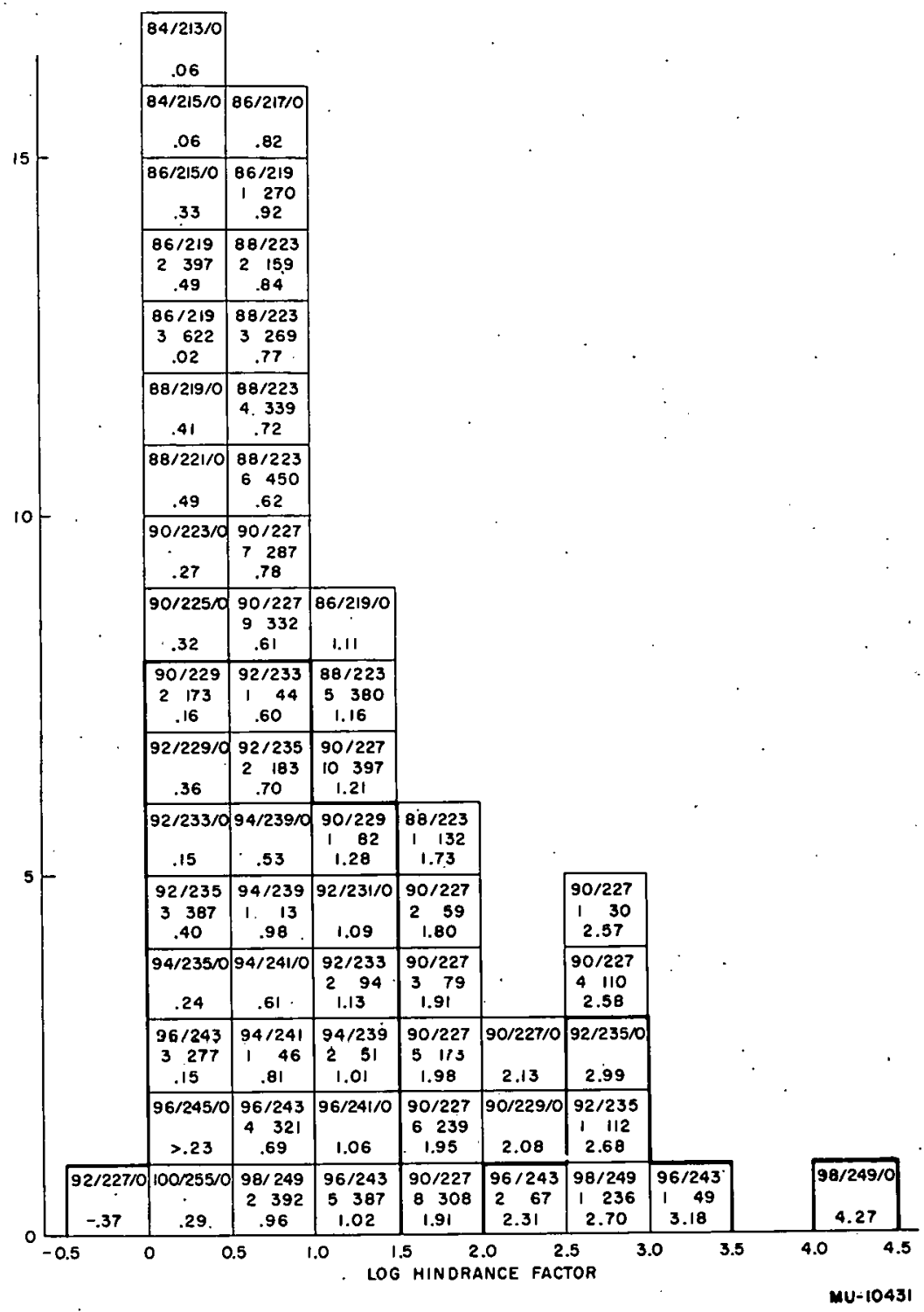

Fig. 9 Distribution of hindrance factors for alpha decay for all observed cases to stales in odd-even nuclei. 


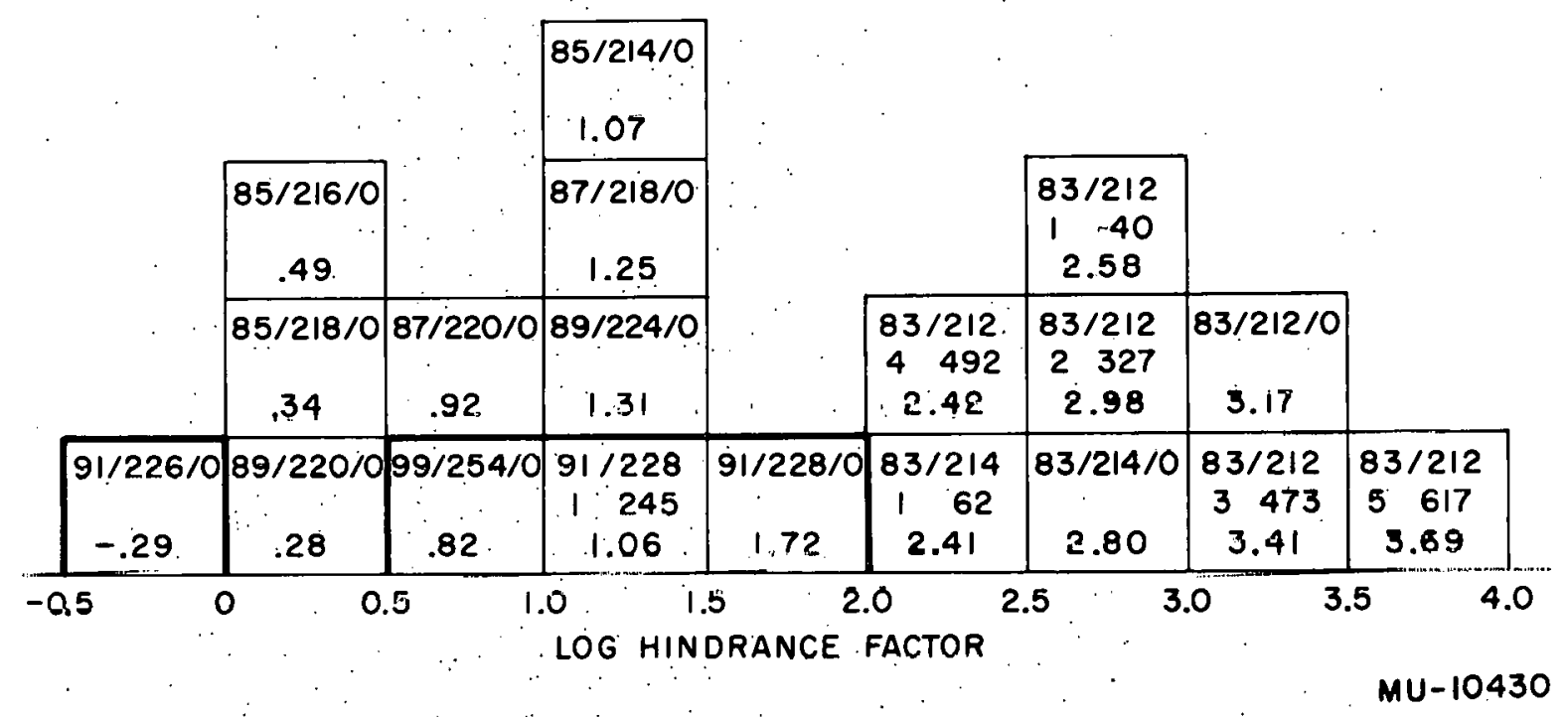

Fig. 10. Distribution of hindrance factors for alpha decay for all observed cases to states in odd-odd nuclei. 